\title{
Magnetic flux ropes: Fundamental structures for eruptive phenomena
}

\author{
Tahar Amari ${ }^{1}$ and Jean-Jacques Aly ${ }^{2}$ \\ ${ }^{1}$ CNRS, Centre de Physique Théorique de l'Ecole Polytechnique, \\ F-91128 Palaiseau Cedex, France \\ email: amari@cpht.polytechnique.fr \\ ${ }^{2}$ AIM - Unité Mixte de Recherche CEA - CNRS - Université Paris VII - UMR n ${ }^{0} 7158$, Centre \\ d'Etudes de Saclay, F-91191 Gif sur Yvette Cedex, France
}

\begin{abstract}
We consider some general aspects of twisted magnetic flux ropes (TFR), which are thought to play a fundamental role in the structure and dynamics of large scale eruptive events. We first discuss the possibility to show the presence of a TFR in a pre-eruptive configuration by using a model along with observational informations provided by a vector magnetograph. Then we present, in the framework of a generic model in which the coronal field is driven into an evolution by changes imposed at the photospheric level, several mechanisms which may lead to the formation and the disruption of a TFR, including the development of a MHD instability, and we discuss the issues of the energy and helicity contents of an erupting configuration. Finally we report some results of a recent and more ambitious approach to the physics of TFRs in which one tries to describe in a consistent way their rising through the convection zone, their emergence through the photosphere, and their subsequent evolution in the corona.
\end{abstract}

Keywords. Flux ropes, Eruptive events, MHD

\section{Why flux ropes?}

Confined eruptive flares, eruptive prominences, coronal mass ejections (CMEs) and interplanetary magnetic clouds (IMCs) are most generally thought to be different observational aspects of a unique phenomenon, the eruptive event. Their respective structures exhibit indeed many similarities. In particular, they quite often show directly or indirectly the presence of a twisted magnetic flux rope (TFR).

Let us recall a few basic observational facts concerning these large scale eruptive phenomena to see how TFR are actually involved (a detailed review of the many observations may be found in Gopalswamy et al. 2006). A typical CME is constituted of a front, a dark cavity, and a plasmoid which contains about $10^{16} \mathrm{~g}$ of material. The latter probably originates from a prominence, i.e., a sheet of relatively cold and dense plasma (compared to the surrounding corona) which may stay in quasi-equilibrium for long periods of time (prominences are highly interesting objects which have been given a great deal of attention by solar physicists; see the review paper by Schmieder in this volume, and Forbes et al. 2006). Such a prominence is often seen indeed to rise before the CME and an associated flare, and it may be naturally thought that it gets ejected with the CME at the average speed of $10^{3} \mathrm{kms}^{-1}$. By simply looking at the images provided by observations one may often guess the presence of twist in an eruptive prominence (as in the well known one called "Granddady"). Moreover, the presence in many cases of a TFR has been much supported by Gary \& Moore (2004) who made a quantitative study 
showing indeed that a twisted structure clearly appears during the eruptive phase (see also Gibson et al. 2006).

In this paper, we report on some particular issues related to the possible role of TFRs in CMEs. We address in particular the following crucial issues: Is a TFR already present in a pre-eruptive configuration, or does it get created during the eruption; does a TFR containing a prominence just traces out the visible CME phenomenon as a passive entity or does it play a role in the initiation of the ejection itself. Thus this is not a review paper on possible CMEs mechanisms. For detailed up-to-date interesting reviews on that more general topics, we refer the readers to , e.g., Priest \& Forbes (2002), Gopalswamy et al. (2006), and Mikic \& Lee (2006).

\section{Flux-Rope and Pre-Eruptive configuration}

Let us start with some general remarks on eruptive phenomena. The first and most important one is that they have to be of magnetic origin. This conclusion appears quite inescapable if we just make a comparison between the various possible sources of energy present in the corona: magnetic, thermal, gravitational, kinetic. Only the first one has a sufficient magnitude to power a CME, say. The second point is that, in the preeruptive phase, the low corona appears to be in quasi-equilibrium, the magnetic, pressure, and gravitational forces balancing each other. In fact, owing to the dominance of the magnetic energy, the equilibrium may be considered to a very good approximation as being forcefree, with the magnetic pressure being thus balanced by the magnetic tension, while the two other forces just intervene to fix the distribution of the plasma along the field lines (Priest \& Forbes 2002). The third point concerns the storage of the energy: It has to be associated with coronal electric currents. In fact the magnetic field $\mathbf{B}$ can be expressed as the sum of a potential term created by the photospheric currents and of a term created by the coronal currents. The magnetic energy is the sum of the energies of these two fields, with only the second one being liable to get dissipated.

Unfortunately, the magnetic field cannot yet be accessed directly in the corona by observational means. One thus needs to build up models to try to understand the details of the processes leading to an eruptive event. Here, we shall restrict our attention to two classes of models which have a long tradition behind them: The TFR model and the Magnetic Arcade (MA) one, which have both developed into a large variety of submodels (Figure 1). The presence of a TFR as shown on the left figure (Amari et al. 2000) is actually a generic feature of a variety of magnetic configurations which have been studied since the middle 80's in the context of solar prominence modelling. The TFR gives indeed to a field the geometric properties needed to ensure the support of cold material, as magnetic dips are obviously present, and a series of models of increasing complexities have been constructed to describe this support, starting with simple pictures in which the prominence is represented by a line current in equilibrium either in a potential field (Anzer \&Priest 1985) or in a linear force-free field (Amari \&Aly 1989), and ending with the much more sophisticated models reported, e.g., in Aulanier \& Demoulin (1998), Titov \& Démoulin (1999), and Lionello et al. (2002). See Forbes et al. (2006) for a more detailed discussion. The arcade model on the right (Antiochos et al. 1999) is also generic of a class of models.

To prove or disprove the existence of one type of structure rather than the other in the pre-eruptive configuration is an important issue which may be solved to some extent by using the measurements performed at the photospheric level by a vector magnetograph, along with a good method to resolve the well-known 180 degrees ambiguity on the transverse field, a problem which has been given recently a great deal of attention 

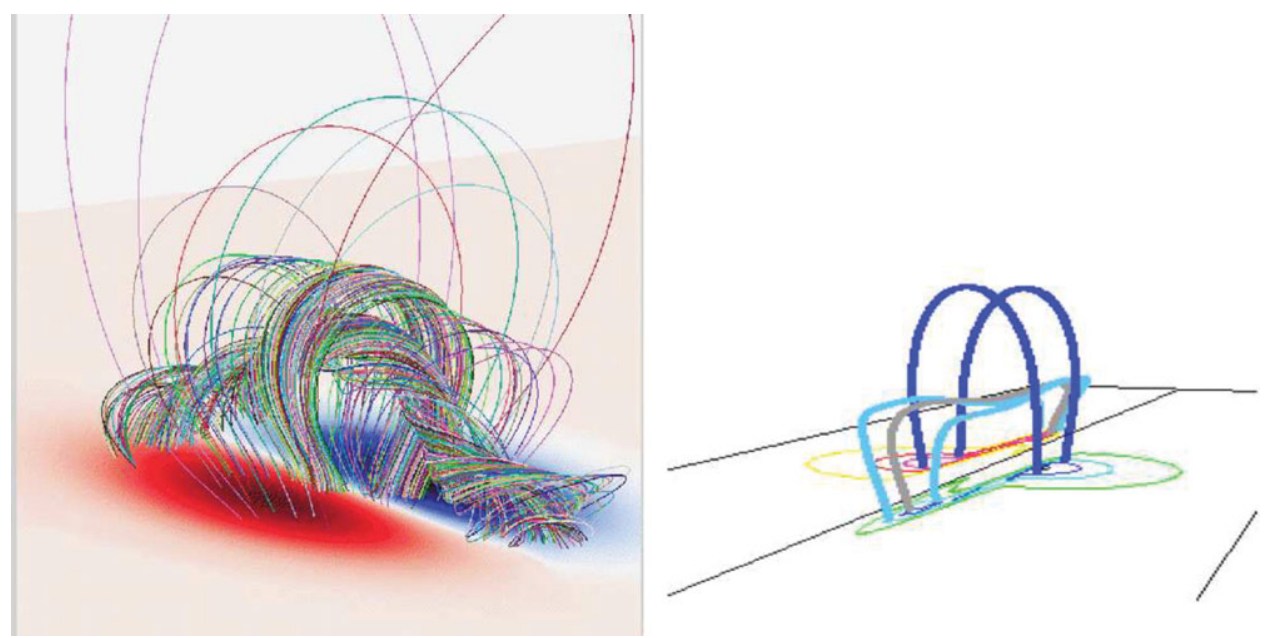

Figure 1. Two generic models which are candidates for describing a pre-eruptive magnetic configuration: On the left, the twisted flux rope from Amari et al. (2000), on the right, arcade model from Antiochos et al.(1999a)

(Li et al. 2007, Metcalf et al. 2006). There seems to be two possible ways to proceed: Either one may merely investigate the orientation of the transverse field, or one may reconstruct the coronal field. The first method rests on the following remark, which has been long pointed out in the context of solar prominences: There must be a reversal from a normal to an inverse type configuration - in which the transverse field points from the negative polarity towards the positive one - if a TFR is present down to the photospheric level as it is the case in some MHD models (Amari et al. 2003a). Although quite simple in principle, this method has a strong limitation: It does not allow to conclude at the absence of a TFR if no one is revealed - e.g., a TFR could exist at higher coronal altitude above a sheared arcade present at the lower level. In the latter case, it is necessary to appeal to the second and much heavier method, which has been much developed in the last few years (Amari et al. 1997, Schrijver et al. 2006, Aly \& Amari 2007, Wiegelmann 2008) in relation with the availability of several ground based (IVM, SOLIS, ASP, THEMIS, EST) and embarked vector magnetographs HINODE, and the prospect of several new ones (SDO, SOLAR-ORBITER) in the near future. Evidences for TFR have thus been obtained in some pre-eruptive configurations by Bleybel et al. (2002), Régnier \& Amari (2004) (see Figure 2), Thalmann \& Wiegelmann (2008), and Canou et al. (2008, in preparation).

\section{Flux Rope and the coronal evolution problem}

Assuming that configurations with TFR do exist, the question of their formation and evolution immediately arises. For about 35 years, this problem has been mainly studied in the framework of a general model in which the solar corona magnetic field is made to evolve in response to changes occuring at the photospheric level, and this has lead to formulating the Coronal Evolution Problem. In the latter one starts from an initial potential or low beta force-free configuration, and makes it evolving by prescribing motions of either one of the following types on the photosphere: Either shearing motions, or flux emergence or submergence, both corresponding to some observations.

When considering a solution to that problem, one has to address the important question of the evolution of two basic quantities: The magnetic energy $W$ - the source powering an 


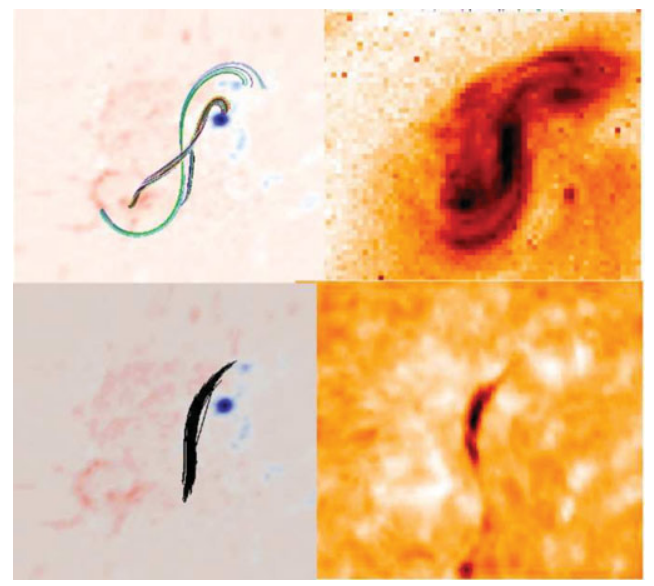

Figure 2. Nonlinear force-free reconstruction of a pre-eruptive configuration from IVM boundary data. Two TFRs may be observed. The first one has a twist exceeding one turn and coincides with the X-ray loops (sigmoid). The second one, located underneath, is less twisted, and its dips (bottom row) support cool material seen in H-alpha line. From Régnier \& Amari (2004)

eruptive event -, and the relative magnetic helicity $H$ (Démoulin 2007, Amari et al. 2003a, Amari et al. 2003b). At least in a nondynamical phase, the evolution of both quantities is essentially controlled by exchanges occurring at the photospheric level (through the Poynting vector for $W$ ). A question which thus naturally arises is that one of the possible existence of upper bounds on the amounts of energy and helicity which can be injected into the field by the photospheric motions.

As for the energy we underline two important theorems which do apply to quasiequilibrium configurations and are thus relevant indeed in a pre-eruptive phase (see Aly \& Amari 2007 for a detailed discussion). They do involve two reference fields having the same distribution of photospheric normal component, $B_{n}$, as $\mathbf{B}$ : The potential field $\mathbf{B}_{\pi}$, and the open field $\mathbf{B}_{\sigma}$, the latter having all its lines being open and thus containing current sheets. Theorem 1 states that the energy $W[\mathbf{B}]$ of a force-free field $\mathbf{B}$ is bounded from below by the energy of $\mathbf{B}_{\pi}$, which justifies the third remark made at the beginning of the previous section. Theorem 2 (Aly 1984) states that $W[\mathbf{B}]$ is bounded from above by a number depending only on $B_{n}$, the best possible upper bound - the so-called least upper bound - being conjectured to be equal to the energy of $\mathbf{B}_{\sigma}$. A weakened form of this guess (Aly 1991, Sturrock 1991), in which one's attention is restricted to configurations having all their lines being connected to the boundary, has been supported by several theoretical arguments and simulations (see however Choe \& Cheng 2002). But a few recent numerical examples of axisymmetric solutions exhibiting one or several TFR disconnected from the boundary have been constructed and found to have an energy exceeding $W\left[\mathbf{B}_{\sigma}\right]$ (Flyer et al. 2005, Hu et al. 2003). This shows that the presence of TFRs can lead to quite large values of the magnetic energy. Note however that no fully $3 \mathrm{D}$ configurations with disconnected TFR have yet been obtained.

That the absolute value of the magnetic helicity of a force-free field may be also bounded from above by a number depending only on $B_{n}$ has been conjectured by Zhang et al. (2006), but no proof of that statement has been yet furnished. If true, this would imply immediately that imparting sufficient shear or twist to a force-free field leads in any case to a nonequilibrium process. 


\section{Flux rope creation and disruption}

In the first class of evolution problems which has been considered by solar physicists, the footpoints of the field lines of an initially potential configuration have been imposed shearing motions, and this problem has been treated both analytically and numerically for fields of increasing geometric complexity: Translation invariant, rotation invariant about an axis (axisymmetric), and more recently fully 3D. In the latter case, the profile of the imposed photospheric flow has been found to be of crucial importance. For a flow exhibiting a strong shear localized near a neutral line, one observes the formation of a sheared arcade in equilibrium when the field topology is bipolar, with no disruption occuring (Antiochos et al. 1999; in the corresponding 2D situation a plasmoid is ejected when a small resistivity is introduced (Amari et al. 1996a)). On the contrary, when the flow leads to a global twisting of the field (which is equivalent to a shearing only near the neutral line), the configuration evolves slowly through a sequence of quasi-equilibria up to a certain twist threshold of about 1 turn. Once the latter is exceeded, the configuration experiences a transition towards a dynamic evolution. A central flux rope is created, which pierces through the overlying field lines and erupts, but without disconnecting from the photosphere. This phenomenon has been called very fast opening (Amari et al. 1996b) and it has been revisited more recently by Török \& Kliem (2003) and Aulanier et al. (2005). Several features of this model may be related to observational facts. In particular the existence of strong electric currents localized below the flux rope may explain the well known characteristic sigmoidal structures. The fact that the TFR remains attached to the Sun while expanding in the solar wind and the interplanetary medium may explain why it may look open from the low corona point of view while appearing still closed in the interplanetary medium where it may be possibly identified as a magnetic cloud (Démoulin 2008). Moreover the interaction of this expanding TFR with the overlying field, which leads to the appearance of strong currents at the interface, may be at the origin of EIT waves as recently proposed in Delannée \& Amari (2000) and Delannée et al. (2008). Finally we point out that the very fast opening phenomenon involves here a partial opening rather than a total one (the open field conjecture is then not challenged). Therefore a full opening is not necessarily implied in a disruption (Amari et al. 1996b).

More recently the effects of photospheric flux changes have also been considered. They may mimic the emergence or submergence of flux through the photosphere, and in particular the so-called flux cancellation process (FC). The latter is often observed on the Sun and it has been given a great deal of attention after Martin et al. (1985). It has been found for instance to occur in the big X 5.7 "Bastille day" event in 2000 (Kosovichev \& Zharkova 2001). Originally proposed as a mechanism leading to the formation of a prominence inside a TFR contained in a 2D equilibrium (van Ballegooijen \& Martens 1989), and also to the formation of an erupting plasmoid in an axisymmetric configuration (Forbes \& Priest 1995), FC has been studied in 3D (Amari et al. 2000, Linker et al. 2001) as a possible process leading to the creation and the disruption of a TFR. If one starts from an initial sheared configuration containing a non-zero magnetic helicity, FC leads after a certain threshold to the creation of a TFR in equilibrium, which experiences later on a major global disruption. The key point here is that there is a decrease of the energy of the open field (which depends only on the photospheric distribution of the normal field component) while the energy of the evolving low beta coronal configuration (which is related to the presence of coronal currents) does not change significantly. Thus both these energies become comparable at some critical time, which precludes the existence of a global equilibrium and leads to the disruption (Amari et al. 2000). The TFR created by the FC mechanism may possibly explain several observed characteristics 
such as the presence of a prominence (there are dips), the presence of a sigmoid, and the current sheet/cusp formation below the ejected rope.

Another mechanism associated with a flux change on the boundary has been proposed for explaining the following fact. During the death of an active region due to the dispersion of its flux, large scale eruptive events are nonetheless produced and reformation of filaments from remnants of previous eruptions are observed. Following Leighton (1964) and Wang et al. (1991), this dispersion has been modelled by turbulent diffusion (TD) occuring at the photosheric level (Amari et al. 1999, Amari et al. 2003b). This leads once more to a well defined BVP in which one starts from an initial configuration supposed to represent the remnant of a previous eruptive field which has relaxed to a non-potential configuration and thus has a nonzero helicity. The field is thus made to evolve slowly due to TD, and it is found that in all cases the resulting evolution leads to the formation of a TFR in equilibrium. Depending on the initial helicity contents, either a confined disruption (moderate helicity) or a global one (large enough helicity) is produced eventually. Although this could seem to show that a minimum amount of magnetic helicity be necessary to trigger a CME, say, it should be noted that the total magnetic helicity of the configuration remains unchanged during the evolution. Once more, the results of this model are in agreement with several observational characteristics of eruptive events.

TFR have also been shown to form when the evolution is driven by converging motions applied to an initial configuration with a non-zero helicity. This problem has been first considered in 2D (Priest \& Forbes 1990) and more recently in 3D by Amari et al. (2003a). By starting from the set of initial configurations previously used in the FC studies, it has been shown that the field evolves through a series of equilibria up to a certain threshold beyond which the topology changes to a TFR-like one. However unlike in the FC or TD mechanisms the TFR is not in equilibrium, and it experiences a disruption. Here TFR formation and disruption appears to be associated.

\section{Flux ropes and robustness with respect to magnetic topology}

As indicated above a very localized shear applied to a simple topology bipolar configuration does not lead to a disruption. The situation turns out to be quite different, however, if the bipolar configuration is a part of a larger quadrupolar configuration. Taking such a complex topology field as an initial state in the BVP previously solved for the simple bipole, it is found indeed (Antiochos et al. 1999) that there is formation in a first stage of a strongly sheared arcade with dips favorable to prominence support. Beyond a critical threshold the field lines above the coronal X-point reconnect with the inner bipolar lines, thus triggering a large scale disruption. For this mechanism to be efficient, it is necessary that the current sheet which forms near the location of the initial X-point be maintained in equilibrium all along the first part of the evolution for otherwise only an insufficient amount of free energy would be stored (note that describing a current sheet is numerically difficult). This interesting mechanism is called the Break out Model $(\mathrm{BOM})$, and it has the merit of showing the role of the magnetic topology in an evolution. It should be noted, however, that this role was also pointed out in earlier $2 \mathrm{D}$ studies (Forbes \& Isenberg 1991, Isenberg et al. 1993).

Some observations have shown that several pre-eruptive configurations had a complex topology (this was the case in particular for the July 14, 1998 flare, as found by Aulanier et al. 2000), and one could be tempted to take this fact as an evidence in favor of the only BOM (Lynch et al. 2008; see Figure 3). However, that a disruption occurs when the field has a complex topology is not the signature of a particular mechanism, it is just one component of the context in which the BOM may be relevant. In fact, if one 

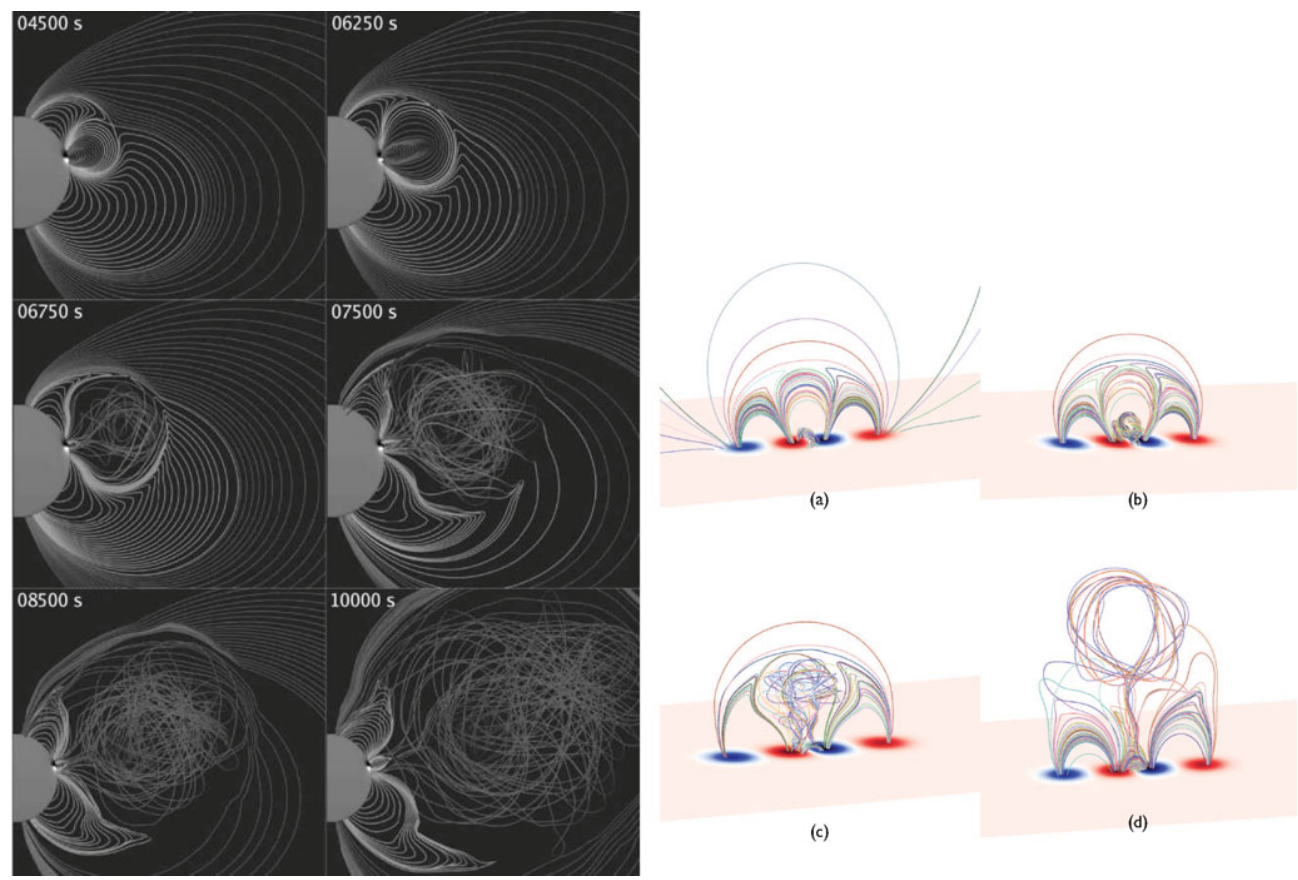

Figure 3. Twisted flux Ropes created in the BOM and in the FC model. In the BOM (left panel, from Lynch et al. 2008), which presupposes a complex topology, the TFR is created during the eruptive phase, while in the FC model (right panel, from Amari et al. 2007) the TFR is formed prior to the eruption as an equilibrium structure existing independently of the nature (bipolar or multipolar) of the background configuration.

takes a quadrupolar configuration as the one used in the BOM, and submit it to FC, then it is found that a TFR in equilibrium gets formed in a first phase in the inner bipolar part. A disruption is thus suffered by the configuration in a second phase (Amari et al. 2007). Compared to the case of a simple dipolar configuration, it is clear that the overlying arcade has weaker confinement properties due to the presence of the Xpoint, which allows a faster expulsion of the TFR. To conclude this section, we note that the BOM and the FCM share the properties of weakening the confinement, and of producing a TFR. But they involve different processes and different structures in the lower part of the magnetic configuration, the TFR appearing before the eruption as an equilibrium structure in the FCM, while it is created in the BOM by a nonequilibrium process involving a shear transfer by reconnection between two initially disconnected topological cells.

\section{Flux ropes prone MHD instabilities}

As we have seen previously, a TFR may be produced in an evolving equilibrium which may thus get disrupted at some stage. Basically there are three possibilities to explain this disruption: i) there exists no equilibrium compatible with the photospheric changes as in the FC mechanism; ii) an equilibrium compatible with the photospheric changes may exist but it is too far to be reached (very fast opening mechanism), and iii) an equilibirum exists but it is unstable. We now explore this last possibility in the context of ideal MHD, which may be used here because of the very high conductivity of the low coronal plasma. We first note that simple $2 \mathrm{D}$ arcades have never been found to be 
unstable, and that there is in 3D a known sufficient condition for a force-free equilibrium to be stable: It is that $\alpha L \lesssim 1$ (Aly 1990), where $L$ is the typical length scale of the structure and $\alpha$ the order of magnitude of the force-free function $(\nabla \times \mathbf{B}=\alpha \mathbf{B})$. But no sufficient condition of instability seems to have been established yet.

What about configurations containing TFR? Some informations can be drawn here from the many studies of cylindrical and toroidal configurations which have been conducted up to now in the context of thermonuclear fusion in magnetically confined plasma. For instance, cylindrical and toroidal TFR configurations have long been shown by plasma laboratory physicists to be subject to the kink instability when the poloidal component of the magnetic field becomes of the order of the axial one (Freidberg 1987). Solar physicists have thus looked for the possibility of the development of the kink in a cylindrical coronal TFR exhibiting a twist of about one turn around the axis when the anchoring of the footpoints in two horizontal plates representing the dense photosphere is taken into account (Raadu 1972, Baty \& Heyvaerts 1996, Baty 1997). The more realistic case of a toroidal line-tied field has also been studied, mainly by considering the 2D analytical model of Titov \& Démoulin (1999), and kink unstable TFR have been obtained in spite of the stabilizing line-tying effect (Török et al. 2004). This mechanism may reproduce some of the observed characteristics of confined disruptions (Török \& Kliem 2005, Fan \& Gibson 2004, Fan \& Gibson 2007). And finally similar conclusions have been suggested by the results of some non-symmetric simulations (Amari \& Luciani 1999).

Another property of TFR discovered by studying fusion toroidal configurations is the fact that a poloidal magnetic field exerts a net outward radial force par unit of length on the toroidal current as a simple consequence of flux conservation (Freidberg 1987). In a tokamak there exist some restoring forces due either to the presence of a wall, which induces a restoring pressure build up in the external part, or to an external vertical field $\mathbf{B}_{\text {ext }}$. In the latter case, however, a too fast decrease of $\mathbf{B}_{\text {ext }}$ with the distance to the axis makes the resulting equilibrium unstable. This is the so-called torus instability, and it has been suggested that it could also occur in the solar corona. In that case, the "external" magnetic field is the one of the overlying arcade, and it has been proven indeed that the torus instability may develop for some shape of the inner TFR and some decreasing profile of $\mathbf{B}_{\text {ext }}$, with a coronal disruption thus being produced (Kliem \& Török 2006).

It is worth noticing that although both the kink and the torus instabilities are interesting exact properties of TFR, their application to the disruption at the origin of eruptive events is not yet completely convincing. In their simple form used up to now, they do develop indeed in a pre-eruptive configuration with a high degree of symmetry. The latter then exhibits the well known phenomenon of symmetry breaking once 3D perturbations are allowed. Such a symmetry certainly exists for a laboratory device like a tokamak, but it seems quite difficult to think of a consistent mechanism which could produce a similarly constrained equilibrium in the solar corona. In fact it should be clear from the results above that a low beta symmetric configuration, once twisted or subject to flux changes, evolves quite generally into a non-symmetric state. Moreover it may be noticed that although these models are able to produce interesting quantitative predictions for the acceleration profiles in CMEs, they may disagree with some recent observations (Schrijver et al. 2008).

\section{Flux ropes driven from below: Towards a global approach}

As stated in Sect. 3, the evolution of the solar corona magnetic field has long been studied as a BVP in which an initial equilibrium is driven into an evolution by prescribing some specific changes in the photospheric parameters. TFRs have thus been shown to 
be created, e.g., by FC, which can be considered in some sense as mimicking the late phase of TFR emergence. This is clearly why the created TFR does not depend on the overlying configuration, which may or may not have a complex topology. To go a step further towards a realistic model, it is clear that one should add a description of the actual process of emergence of the TFR from the convection zone (CZ) into the corona.

The most ambitious approach to try to deal with such a program consists in setting at once a global MHD model including both the $\mathrm{CZ}$ and the corona, which constitutes a huge numerical challenge because of the strong difference in the plasma characteristics in both regions, and the stiff variations in the photospheric transition. In spite of that, this formidable task has yet been faced by several authors (Fan 2001, Archontis et al. 2004, Magara \& Longcope 2003, Manchester et al. 2006, Galsgaard et al. 2005, Cheung et al. 2007). However, although this global approach has to be taken eventually, it may be useful in a first step to try to gain some simple insights into the complicated physics involved in that problem by following a more modest but complementary approach in which an initial TFR is introduced in the $\mathrm{CZ}$ and forced to rise rigidly. This leads once more to solving a coronal BVP as the one driven by photospheric changes. But the imposed changes are now those which generate an electric field associated to a TFR that is vertically crossing the photosphere at a given velocity. In the $\mathrm{CZ}$, the TFR can be taken to be either a torus rising at a uniform speed (Fan \& Gibson 2004, Fan \& Gibson 2007), or a cylinder deformed by an horizontally varying vertical flow, which makes only the central part to "emerge" (Amari et al. 2004). In both cases its confinement in the CZ is insured by the external high pressure field (it has not to be assumed to be force-free). This leads to the emergence of the TFR into the low beta corona, with a nonequilibrium behavior occurring eventually or not depending on the amount of initial twist and on the strength of the coronal confinement. It is worth noticing that this simple model reproduces the characteristic features of the so-called "tongs" which have been observed in the concentration of the photospheric normal component of the field (López Fuentes et al. 2000) and were already seen in earlier MHD simulations (Fan 2001).

Although reproducing many observed facts, the approach above has some heavy shortcomings. In particular theoretical arguments and numerical simulations show that a TFR rigidly rising in the $\mathrm{CZ}$ converts a part of its vertical motion into horizontal one when hitting the photosphere. This of course prevents its rapid emergence, which is further impeded by the accumulation of heavy material in the dips. A possible way to bypass these problems consists in transferring the horizontal component of the magnetic field, which is responsible for the normal component of the electric current, before the vertical rising flow in the CZ becomes too small. That is what is done in the Resistive Layer Model (RLM) (Amari et al. 2005) in which the turbulent photospheric transition is represented by a resistive layer. An important feature of that model is that it insures the conservation of the global magnetic helicity. In fact helicity injection through the interface depends only on the tangential component of the electric field and the normal component of the magnetic field, and the latter are in any case always continuous. When one goes from the $\mathrm{CZ}$ to the corona, the term in the Ohm's law which is dominant in the determination of the horizontal electric field just changes. Below, it is the effect of the vertical component of the flow (and the horizontal component of the magnetic field) which is important, thus it is the magnetic diffusivity (and the electric current), and finally it is the shear flow, say, (and the vertical component of the magnetic field) which becomes important at the basis of the corona (a remaining vertical flow may of course be added to that horizontal flow). The RLM has been shown to allow indeed the emergence of the TFR into the corona (while no emergence occurs if the resistivity is made to vanish), followed later on 
by its disruption (Amari et al. 2005). The difference with the rigid case considered above is that magnetic helicity does keep increasing but tends to saturate.

\section{Conclusion}

Magnetic flux ropes are structures which may be easily formed in the solar corona by various mechanisms. They are good candidates to support prominence material which is denser and cooler than the coronal one around. They may lead to either confined disruption or large scale eruptive phenomena such as CMEs and two ribbon flares. Their interest also relies on the fact that they may be subject to various ideal instabilities such as the kink and the torus instabilities. There are several indications that they may be present in the corona prior to some eruptive event. In particular, this has been shown to be true in some cases by using boundary data provided by vector magnetographs and a force-free low corona model, without then making any extra assumptions on their origin. Considering only MHD mechanisms, TFR have been shown either to exist in the preeruptive configuration or to be created only during the eruption through reconnection. They may also represent the magnetic structure of interplanetary magnetic clouds. Of course we do not mean that TFRs are the only way of triggering eruptive phenomena, and we acknowledge the fact that many other types of structures may produce such events. Determining if TFR may also come from below is one of the main challenge of current research in solar physics. Answering this question requires the construction of a model allowing to follow TFR from their possible formation in the stable region below the $\mathrm{CZ}$, their rising through the latter, their piercing through the photosphere, and their evolution in the corona. Much help should be also provided in this respect by the arrival of a new generation of vector magnetographs with low noise and high resolution such as those on board of HINODE or SDO or the future ground based EST.

\section{References}

Aly, J. J. 1984, Astrophys. J., 283, 349

Aly, J. J. 1990, Physics of Fluids B, 2, 1928

Aly, J. J. 1991, Astrophys. J., 375, L61

Aly, J. J. \& Amari, T. 2007, Geophysical and Astrophysical Fluid Dynamics, 101, 249

Amari, T. \& Aly, J. J. 1989, Astron. Astrophys., 208, 261

Amari, T., Luciani, J. F., Aly, J. J., \& Tagger, M. 1996, Astron. Astrophys., 306, 913

Amari, T., Luciani, J. F., Aly, J. J., \& Tagger, M. 1996, Astrophys. J., 466, L39

Amari, T., Aly, J. J., Luciani, J. F., Boulmezaoud, T. Z., \& Mikic, Z. 1997, Solar Phys., 174, 129

Amari, T. \& Luciani, J. F. 1999, Astrophys. J., 515, L81

Amari, T., Luciani, J. F., Mikic, Z., \& Linker, J. 1999, Astrophys. J., 518, L57

Amari, T., Luciani, J. F., Mikic, Z., \& Linker, J. 2000, Astrophys. J., 529, L49

Amari, T., Luciani, J. F., Aly, J. J., Mikic, Z., \& Linker, J. 2003, Astrophys. J., 585, 1073

Amari, T., Luciani, J. F., Aly, J. J., Mikic, Z., \& Linker, J. 2003, Astrophys. J., 595, 1231

Amari, T., Luciani, J. F., \& Aly, J. J. 2004, Astrophys. J., 615, L165

Amari, T., Luciani, J. F., \& Aly, J. J. 2005, Astrophys. J., 629, L37

Amari, T., Aly, J. J., Mikic, Z., \& Linker, J. 2007, Astrophys. J., 671, L189

Antiochos, S. K., Devore, C. R., \& Klimchuk, J. A. 1999, Bulletin of the American Astronomical Society, 31, 868

Antiochos, S. K., DeVore, C. R., \& Klimchuk, J. A. 1999, Astrophys. J., 510, 485

Anzer, U. \& Priest, E. 1985, Solar Phys., 95, 263

Archontis, V., Moreno-Insertis, F., Galsgaard, K., Hood, A., \& O'Shea, E. 2004, Astron. Astrophys., 426, 1047 
Aulanier, G. \& Demoulin, P. 1998, Astron. Astrophys., 329, 1125

Aulanier, G., Démoulin, P., \& Grappin, R. 2005, Astron. Astrophys., 430, 1067

Aulanier, G., DeLuca, E. E., Antiochos, S. K., McMullen, R. A., \& Golub, L. 2000, Astrophys. J. , 540, 1126

Baty, H. \& Heyvaerts, J. 1996, Astron. Astrophys., 308, 935

Baty, H. 1997, Astron. Astrophys., 318, 621

Bleybel, A., Amari, T., van Driel-Gesztelyi, L., \& Leka, K. D. 2002, Astron. Astrophys., 395, 685

Cheung, M. C. M., Schüssler, M., \& Moreno-Insertis, F. 2007, Astron. Astrophys., 467, 703

Choe, G. S. \& Cheng, C. Z. 2002, Astrophys. J., 574, L179

Delannée, C. \& Amari, T. 2000, Bulletin of the American Astronomical Society, 32, 838

Delannée, C., Török, T., Aulanier, G., \& Hochedez, J.-F. 2008, Solar Phys., 247, 123

Démoulin, P. 2007, Advances in Space Research, 39, 1674

Démoulin, P. 2008, Ann. Geophys., in press

Fan, Y. 2001, Astrophys. J., 554, L111

Fan, Y. \& Gibson, S. E. 2004, Astrophys. J., 609, 1123

Fan, Y. \& Gibson, S. E. 2007, Astrophys. J., 668, 1232

Flyer, N., Fornberg, B., Thomas, S., \& Low, B. C. 2005, Astrophys. J., 631, 1239

Forbes, T. G. \& Isenberg, P. A. 1991, Astrophys. J., 373, 294

Forbes, T. G. \& Priest, E. R. 1995, Astrophys. J., 446, 377

Forbes, T. G., Linker,J. A., Chen, J., Cid, C., Chen, J.,Kóta, J., Lee, M. A., Mann, G., Mikić, Z. , Potgieter, M. S., Schmidt, J. M. , Siscoe, G. L., Vainio, R., Antiochos, S. K., \& Riley, P. 2006, Space Science Reviews, 123, 251

Freidberg, J. P. 1987, Ideal Magnetohydrodynamics. Plenum Press, New York

Galsgaard K., Moreno-Insertis F., Archontis V. \& Hood A. 2005, ApJL, 618, L153

Gary, G. A. \& Moore, R. L. 2004, ApJ, 611, 545

Gopalswamy, N. , Mikić, Z., Maia, D., Alexander, D., Cremades, H., Kaufmann, P., Tripathi, D., \& Wang, Y.-M. 2006, Space Science Reviews, 123, 303

Gibson, S. E., Fan, Y., Török, T., \& Kliem, B. 2006, Space Science Reviews, 124, 131

Hu, Y. Q., Li, G. Q., \& Xing, X. Y. 2003, Journal of Geophysical Research (Space Physics), 108, 1072

Isenberg, P. A., Forbes, T. G., \& Demoulin, P. 1993, Astrophys. J., 417, 368

Kliem, B. \& Török, T. 2006, Physical Review Letters, 96, 255002

Kosovichev, A. G. \& Zharkova, V. V. 2001, Astrophys. J., 550, L105

Leighton, R. B. 1964, Astrophys. J., 140, 1547

Li, J., Amari, T., \& Fan, Y. 2007, Astrophys. J., 654, 675

López Fuentes, M. C., Demoulin, P., Mandrini, C. H., \& van Driel-Gesztelyi, L. 2000, Astrophys. $J ., 544,540$

Lynch, B. J., Antiochos, S. K., DeVore, C. R., Luhmann, J. G., \& Zurbuchen, T. H. 2008, Astrophys. J., 683, 1192

Linker, J. A., Lionello, R., Mikić, Z., \& Amari, T. 2001, J. Geophys. Res., 106, 25165

Lionello, R., Mikić, Z., Linker, J. A., \& Amari, T. 2002, Astrophys. J., 581, 718

Magara, T. \& Longcope, D. W. 2003, Astrophys. J., 586, 630

Manchester,W. IV., Gombosi, T., DeZeeuw D., \& Fan Y. 2006, Astrophys. J., 610, 161

Martin, S. F., Livi, S. H. B., \& Wang, J. 1985, Australian Journal of Physics, 38, 929

Metcalf, T. R., et al. 2006, Solar Phys., 237, 267

Mikić, Z. \& Lee,M. A. 2006, Space Science Reviews, 123, 57

Ott, U. 1993, Nature, 364, 25

Priest, E. R. \& Forbes, T. G. 1990, Solar Phys., 126, 319

Priest, E. R. \& Forbes, T. G. 2002, Astron Astrophys Rev, 10,313

Raadu, M. A.1972, Solar Phys., 22, 425

Régnier, S. \& Amari, T. 2004, Astron. Astrophys., 425, 345

Schrijver, C. J., et al. 2006, Solar Phys., 235, 161

Schrijver, C. J., Elmore, C., Kliem, B., Török, T., \& Title, A. M. 2008, Astrophys. J., 674, 586

Sturrock, P. A. 1991, Astrophys. J., 380, 655 
Thalmann, J. K. \& Wiegelmann, T. 2008, Astron. Astrophys., 484, 495

Titov, V. S. \& Démoulin, P. 1999, Astron. Astrophys., 351, 707

Török, T. \& Kliem, B. 2003, Astron. Astrophys., 406, 1043

Török, T., Kliem, B., \& Titov, V. S. 2004, Astron. Astrophys., 413, L27

Török, T. \& Kliem, B. 2005, Astrophys. J., 630, L97

van Ballegooijen, A. A. \& Martens, P. C. H. 1989, Astrophys. J., 343, 971

Wang, Y.-M., Sheeley, N. R., Jr., \& Nash, A. G. 1991, Astrophys. J., 383, 431

Wiegelmann, T. 2008, Journal of Geophysical Research (Space Physics), 113, 3

Zhang, M., Flyer, N., \& Low, B. C. 2006, Astrophys. J., 644, 575 